Agronomía Costarricense 40(2): 63-75. ISSN:0377-9424 / 2016

www.mag.go.cr/rev_agr/index.html www.cia.ucr.ac.cr

\title{
RESPUESTA DEL PASTO ALPISTE (Phalaris arundinacea L.) A LA FERTILIZACIÓN NITROGENADA EN COSTA RICA
}

Luis Villalobos*

Palabras clave: Biomasa; fenología; fertilización nitrogenada; Phalaris.
Keywords: Biomass; phenology; nitrogen fertilization; Phalaris.

Recibido: 20/01/16

Aceptado: 04/06/16

\section{RESUMEN}

El nitrógeno es el nutriente de mayor importancia en la producción de pasturas y las dosis de fertilización utilizadas deben optimizar la producción de biomasa de alto valor nutricional con un bajo costo y mínima afectación al medio ambiente. Se realizó un estudio con pasto alpiste (Phalaris arundinacea L.) cosechado cada 70 días en una finca comercial productora de leche ubicada en el distrito de Santa Rosa de Oreamuno, en la provincia de Cartago entre noviembre de 2010 a enero de 2012. Se aplicó 3 dosis de fertilización nitrogenada (100, 200 y $300 \mathrm{~kg} \cdot \mathrm{ha}^{-1}$ ) y se evaluó su respuesta productiva en biomasa y valor nutricional por medio de un diseño de bloques completos al azar. La producción de materia seca no fue estadísticamente diferente $(\mathrm{p} \geq 0,05)$ entre las dosis menor y mayor (2956 y $3347 \mathrm{~kg} \cdot \mathrm{ha}^{-1}$, respectivamente), mientras que mostró valores menores y mayores $(\mathrm{p} \leq 0,0001)$ entre los muestreos de febrero y abril (1632 y $4323 \mathrm{~kg} \cdot \mathrm{ha}^{-1}$, respectivamente). Los rangos de concentraciones de proteína cruda $(14,62-$ $19,20 \%)$, fibra detergente neutro $(54,50-70,70 \%)$ y energía neta de lactancia $(1,14-1,32$ Mcal.kg-1 MS) fueron el resultado de los cambios significativos ( $\mathrm{p} \leq 0,0001)$ que se presentaron a lo largo del año en el alpiste forrajero. Estrategias de ajustes en carga animal y suplementación del ganado

Universidad de Costa Rica, Centro de Investigaciones en Nutrición Animal y Escuela de Zootecnia,

\begin{abstract}
Reed canarygrass (Phalaris arundinacea L.) response to nitrogen fertilization rates in Costa Rica. Nitrogen is the most important nutrient in pasture production and the use of fertilizer rates should optimize biomass yield of high nutritional value with low cost and minimum impact to the environment. A study was performed with reed canarygrass (Phalaris arundinacea L.) harvested every 70 days in a commercial dairy farm located in the district of Santa Rosa of Oreamuno, in the province of Cartago from November 2010 to January 2012. Three nitrogen doses were applied (100, 200 and $300 \mathrm{~kg} \cdot \mathrm{ha}^{-1}$ ), and biomass production response and nutritional value through a completely randomized block design was evaluated. Dry matter production was not statistically different $(\mathrm{p} \geq 0.05)$ between the lowest and highest doses (2956 and $3347 \mathrm{~kg} \mathrm{ha}^{-1}$, respectively), while it showed lower and higher values $(\mathrm{p} \leq 0.0001)$ for samplings between February and April (1632 and $4323 \mathrm{~kg} \cdot \mathrm{ha}^{-1}$, respectively). Ranges in crude protein concentration (14.62 to $19.20 \%$ ), neutral detergent fiber (54.50 to $70.70 \%)$ and net energy of lactation (1.14 to $1.32 \mathrm{Mcal}^{\mathrm{kg}} \mathrm{kg}^{-1} \mathrm{MS}$ ) were the result of significant changes $(\mathrm{p} \leq 0.0001)$ that occurred throughout the year in the reed canarygrass. Strategies to adjust stocking rate
\end{abstract}

Costa Rica. Correo electrónico: luis.villalobosvillalobos@ucr.ac.cr 
lechero deben considerarse para llenar las limitantes estacionales encontradas en la biomasa y el valor nutricional del pasto alpiste durante el año.

\section{INTRODUCCIÓN}

Con excepción de la humedad y la temperatura, el nitrógeno es considerado el principal factor que influye sobre el crecimiento de pastos de clima templado (Brink y Casler 2009) debido al impacto potencial que tiene en la producción de biomasa y su efecto en el valor nutricional del mismo (Funderburg et al. 2012). La aplicación de nitrógeno inorgánico es una práctica agronómica ampliamente utilizada para estimular el crecimiento en pasturas, aunado a que es uno de los principales insumos utilizados en sistemas ganaderos lácteos basados en forraje. Debido a ello, el manejo agronómico racional de las dosis y épocas de aplicación de nitrógeno puede disminuir los costos de producción y el impacto ambiental de los sistemas ganaderos lácteos (Brink y Casler 2009, Jin et al. 2010).

En regiones templadas la producción de pastos tiene una marcada estacionalidad por lo que se acostumbra aplicar dosis altas de nitrógeno con el objetivo de obtener la mayor cantidad de biomasa durante la época de crecimiento (Cherney y Cherney 2005). En regiones altas del trópico la elevación y temperatura permiten establecer pastos perennes de clima templado (Villalobos y Sánchez 2010a) cuya productividad presenta variaciones a lo largo del año, relacionadas principalmente con el régimen de precipitación. Dichas condiciones agroclimáticas hacen que la producción de pastos se mantenga relativamente constante durante el año, lo cual permite fraccionar las dosis de fertilizante nitrogenado de forma and supplementation of dairy cattle should be taken into account in order to fulfilled seasonal limitations encountered in biomass and nutritional value of reed canarygrass during the year.

tal que se optimice la utilización de los nutrientes por la planta (Cherney y Cherney 2005).

El fraccionamiento de las dosis de fertilizante nitrogenado en pastos perennes permite una productividad de biomasa sostenida a lo largo del año en comparación con una única aplicación (Cherney y Cherney 2005). Dosis altas de nitrógeno amoniacal y nítrico son más propensas a generar pérdidas de nutrientes por medio de volatilización y lixiviación, respectivamente (Jin et al. 2010).

Los pastos perennes de clima templado han mostrado una respuesta lineal al incrementar la dosis de nitrógeno (Brink y Casler 2009). En regiones altas del trópico se ha investigado la edad de cosecha del pasto alpiste (Villalobos 2012), pero no existe información acerca de la respuesta en producción de materia seca, valor nutritivo y fenología al incrementar la dosis de nitrógeno. El objetivo de la presente investigación fue estimar la capacidad de producción de biomasa, el contenido nutricional y la fenología del pasto alpiste con 3 dosis de nitrógeno a una edad de cosecha de 70 días a lo largo de un año.

\section{MATERIALES Y MÉTODOS}

El estudio se realizó en una finca comercial productora de leche ubicada en el distrito de Santa Rosa, Oreamuno, Cartago de noviembre de 2010 a enero de 2012 donde se cuenta con pasturas del pasto alpiste. La finca se ubica en la sección Oriental del Valle Central (latitud 0954', longitud $83^{\circ} 49^{\prime}$ ) a una altitud de $2000 \mathrm{msnm}$ 
con influencia climática del Caribe y clasificada como bosque húmedo tropical premontano (Holdridge 1947, citado por Janzen 1991). La precipitación promedio anual es de $2239 \mathrm{~mm}$, la temperatura mínima y máxima promedio son $13,4^{\circ} \mathrm{C}$ y $23,3^{\circ} \mathrm{C}$ respectivamente, el brillo solar tiene en promedio 5,5 horas/día y la velocidad promedio del viento $24,4 \mathrm{~km} / \mathrm{h}$ (IMN 2015). Un estudio previo (Villalobos 2012) sirvió como guía para definir la edad de corte que se utilizó en el presente experimento.

Se realizó una cosecha de uniformización en noviembre de 2010 en el área de estudio con el objetivo de brindar 70 días previos al primer muestreo y se asignaron aleatoriamente un total de 12 parcelas experimentales (área $=9,6 \mathrm{~m}^{2}$ parcela $\left.^{-1}\right)$. Se contó con 3 tratamientos experimentales que consistieron en dosis anuales de nitrógeno $\left(100,200\right.$ y $\left.300 \mathrm{~kg} \mathrm{ha}^{-1}\right)$ con 4 repeticiones. Los tratamientos se escogieron con base en dosis utilizadas en sistemas pastoriles en el trópico (Andrade 2006, Villalobos y Sánchez 2010a y Villalobos y Arce 2013). Todas las parcelas recibieron $\mathrm{P}_{2} \mathrm{O}_{5}$ y $\mathrm{K}_{2} \mathrm{O}$ en dosis de 55 y 18 kg.harespectivamente. Las parcelas se cosecharon cada 70 días a lo largo de un año para un total de 5 cosechas en febrero (Feb-02), Abril (Abr-13), Junio (Jun-22), Agosto (Ago-31) y Noviembre (Nov-11) y 60 muestras analizadas. Cada dosis fue distribuida en 4 aplicaciones realizadas posterior a las primeras 4 cosechas por medio de la técnica de voleo. Se aplicó las dosis de fósforo y potasio a partir de la fórmula de fertilizante 10-30-10 y se completó la dosis de nitrógeno con Urea (46-0-0).

\section{Producción de biomasa y valor nutricional}

Las parcelas fueron cosechadas de forma manual a una altura de corte de $10 \mathrm{~cm}$, se pesó toda la biomasa del área de la parcela en fresco y se tomó una muestra representativa de $1 \mathrm{~kg}$ que se secó a $60^{\circ} \mathrm{C}$ por 48 horas para estimar la producción de materia seca (MS) por hectárea. Se estimó la carga animal instantánea al dividir la producción de biomasa entre un factor de 10 que corresponde al consumo en $\mathrm{kg}$ de MS de una unidad animal equivalente a una vaca de $500 \mathrm{~kg}$ no gestante (Mandaluniz et al. 2005). Dicho valor se normaliza a carga animal estable al dividir entre el número de días de rebrote (descanso) en la pastura.

La muestra tomada en cada parcela se tomó por medio de la técnica de cuarteo, con el objetivo de determinar el contenido de proteína cruda (PC) (AOAC 1990), la pared celular (FDN), la lignina y la digestibilidad "in vitro" de la materia seca (DIVMS) (Van Soest and Robertson 1985). Se determinó el fraccionamiento de la proteína (Licitra et al. 1996) y el contenido de carbohidratos no fibrosos (Van Soest et al. 1991). Los componentes de energía expresados como nutrientes digestibles totales (NDT) y energía neta de lactancia $\left(\mathrm{EN}_{\mathrm{L}} 3 \mathrm{X}\right)$ se estimaron con la metodología del NRC (2001) por medio de los valores de referencia del pasto alpiste de MS, fibra detergente ácida y cenizas $(17,58,34,30$ y $12,95 \%$, respectivamente).

\section{Edad fenológica}

Se evaluó la edad fenológica del pasto alpiste por medio del conteo del número de hojas verdes por rebrote. Para ello se seleccionó aleatoriamente 10 observaciones por parcela para un total de 40 observaciones por tratamiento por muestreo ( $\mathrm{n}=600$ observaciones).

\section{Análisis de la información}

Se utilizó un diseño en bloques aleatorizados completos compuesto de 4 bloques (repeticiones) y 3 tratamientos $\left(100,200\right.$ y $300 \mathrm{~kg} \cdot \mathrm{ha}^{-1}$ N) para un total de 12 parcelas. La información de biomasa, calidad nutricional y edad fenológica se sometió a un análisis de varianza (ANOVA) con medidas repetidas mediante el procedimiento PROC MIXED del paquete estadístico SAS® 9.3 (SAS-Institute, 2011). En dicho modelo se analizó el efecto del tratamiento, la fecha de muestreo y la interacción de ambos. Para controlar el efecto de cosechas previas, el tratamiento se anidó en el factor de parcela. Se estimaron las diferencias significativas $(\mathrm{p}<0,05)$ entre medias de las dosis 
de nitrógeno y las fechas de muestreo para todas las variables en base al error tipo III mediante el procedimiento PROC GLIMMIX de SAS ${ }^{\circledR} 9.3$ (SAS-Institute 2011).

\section{RESULTADOS Y DISCUSIÓN}

\section{Producción de biomasa y fenología}

La producción de biomasa del pasto alpiste fue numéricamente mayor al aumentar la dosis de N, sin embargo, no se encontró una tendencia a incrementar la producción de biomasa de forma significativa $(\mathrm{p}>0,05)$ al incrementar la dosis de fertilización al doble y triple de la dosis menor evaluada. La curva de producción de biomasa fue similar para las 3 dosis a lo largo del año (Figura 1), donde la época fue el factor que mostró diferencias significativas $(\mathrm{p} \leq 0,0001)$ entre los 5 muestreos en el pasto alpiste (Cuadro 1). Durante noviembre a febrero la producción de biomasa fue menor, mientras que entre abril y junio incrementó. La producción de biomasa fue similar a la encontrada por Villalobos (2012) para el mismo pasto sin fertilización (3101 kg.ha-1 MS) pero inferior a la de los pastos kikuyo (Andrade 2006) y ryegrass perenne producidos en zonas altas del trópico (Villalobos y Sánchez 2010a) con dosis de nitrógeno de 174 y 200-250 kg.ha-1 $\mathrm{N}$. La cantidad de $\mathrm{N}$ aplicada a pasturas debe ir en relación con una respuesta productiva que justifique la inversión mayor en insumos y mano de obra, en este estudio no se encontró una producción de biomasa mayor al incrementar la dosis de N. Brink y Casler (2009) encontraron producciones de biomasa similares en pastos de clima templado cuando la dosis de $\mathrm{N}$ se incrementó a más de $225 \mathrm{~kg} \cdot \mathrm{ha}^{-1} \mathrm{~N}$.

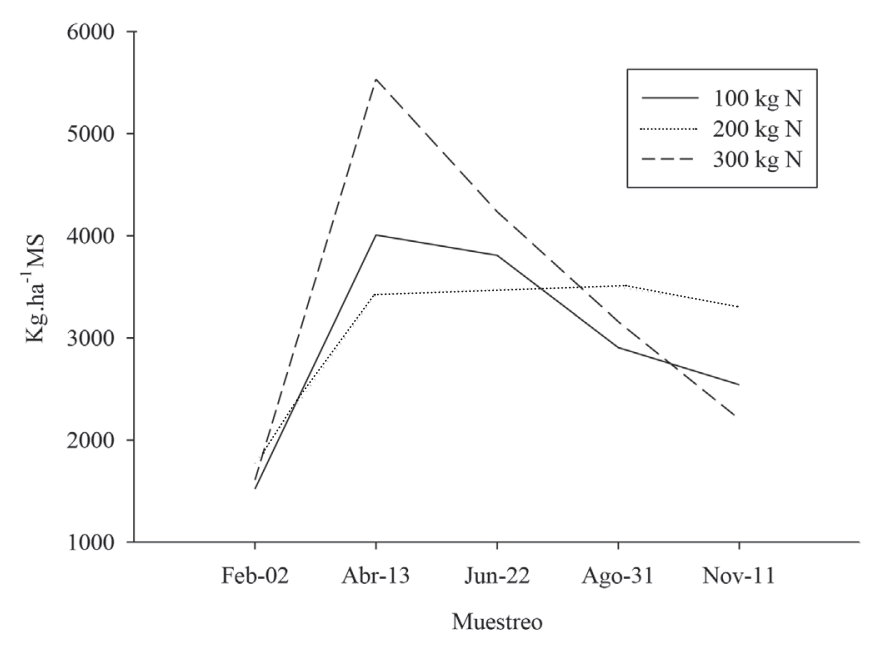

Fig. 1. Comportamiento productivo del pasto alpiste con 3 dosis de $\mathrm{N}$ a lo largo de 5 muestreos en zonas altas del trópico. 
Cuadro 1. Producción de biomasa del pasto alpiste en relación con la fenología a lo largo de un año.

\begin{tabular}{|c|c|c|}
\hline Muestreo $^{1}$ & $\begin{array}{c}\text { Biomasa }^{2} \\
\left(\mathrm{~kg} \cdot \mathrm{ha}^{-1} \cdot \operatorname{cosecha}^{-1} \mathrm{MS}\right)\end{array}$ & $\begin{array}{c}\text { Edad fenológica } \\
\text { (hojas verdes.brote }{ }^{-1} \text { ) }\end{array}$ \\
\hline Feb-02 & $1632^{\mathrm{d}}$ & $5,01^{\mathrm{b}}$ \\
\hline Abr-13 & $4323^{\mathrm{a}}$ & $6,18^{\mathrm{a}}$ \\
\hline Jun-22 & $3840^{\mathrm{ab}}$ & $6,27^{\mathrm{a}}$ \\
\hline Ago-31 & $3186^{\mathrm{bc}}$ & $5,03^{\mathrm{b}}$ \\
\hline Nov-11 & $2684^{c}$ & $4,76^{\mathrm{b}}$ \\
\hline Promedio & 3133 & 5,45 \\
\hline Tratamiento $(\mathrm{T})^{2}$ & 0,7981 & 0,9173 \\
\hline Muestreo (M) & $<0,0001$ & $<0,0001$ \\
\hline $\mathrm{T} * \mathrm{M}$ & 0,2261 & 0,2180 \\
\hline
\end{tabular}

1. Cada muestreo corresponde al promedio de las 12 parcelas.

2. Medias con letras diferentes en una columna difieren entre si según la prueba de Duncan $(\mathrm{p}<0,05)$.

Los programas de fertilización deben considerar los cambios estacionales en producción de biomasa, de forma tal que la aplicación de $\mathrm{N}$ coincida con las épocas cuando las pasturas tienen un potencial productivo mayor. Cherney y Cherney (2005) encontraron producciones acumuladas de biomasa mayores al fraccionar una dosis de $\mathrm{N}$ de $225 \mathrm{~kg} \cdot \mathrm{ha}^{-1} \mathrm{~N}$ en 2 o 3 aplicaciones versus una sola aplicación en 3 pastos perennes (Orchargrass [Dactylis glomerata L.], alpiste forrajero y festuca [Festuca arundinaceae]). Debido a las variaciones estacionales en producción de biomasa mostradas por el pasto alpiste, el programa de fertilización utilizado por los productores lácteos debe optimizar dichas variaciones con el objetivo de aprovechar los nutrientes en el suelo y reducir la potencial emisión de gases de efecto invernadero (Jin et al. 2010).

Similar a la producción de biomasa, la fenología del pasto alpiste varió en relación con la época de muestreo $(\mathrm{p} \leq 0,0001)$ mientras que la dosis de $\mathrm{N}$ aplicada no produjo cambios en el número de hojas verdes por brote (Cuadro 1). Sin embargo, la edad fenológica aumentó en abril y junio, época durante la que se obtuvo la mayor cantidad de biomasa. La mayor parte de las observaciones de edad fenológica (88\%) se ubicó entre 4 y 7 hojas verdes.brote $^{-1}$ (Figura 2) lo cual coincide con lo encontrado por Villalobos (2012) como un rango adecuado para optimizar la producción de biomasa del pasto alpiste. 


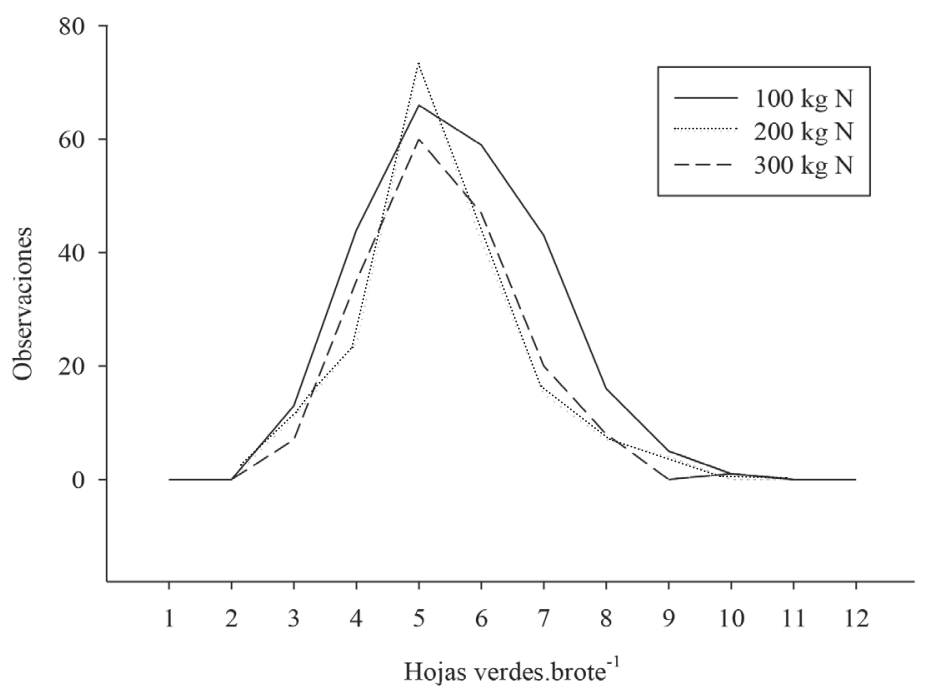

Fig. 2. Distribución de la frecuencia de observaciones del número de hojas verdes por brote del pasto alpiste con 3 dosis de N.

La edad fenológica en pastos se encuentra relacionada directamente con el estado de reservas en la planta y su ciclo de vida. Las hojas producidas en cada brote de pasto cumplen un ciclo de vida luego del cual se convierten en material senescente (Donaghy y Fulkerson 2002). La edad de cosecha de 70 días de rebrote permitió mantener una fenología relativamente constante en el pasto alpiste con 3 dosis de N, por tanto el manejo agronómico (rotación, fertilización, etc.) tiene un efecto menor sobre el número de hojas verdes por brote que la planta produce.

La producción de biomasa promedio por cosecha y anual fue similar $(\mathrm{p}>0,05)$ entre las dosis de $\mathrm{N}$ evaluadas (Cuadro 2). La producción anual del pasto alpiste fue superior a la reportada por otros investigadores en condiciones de clima templado con producciones máximas de 9420 kg.ha' ${ }^{-1}$ y 7300 kg.ha-1 (Geber 2002 Brink y Casler 2009). Lo anterior puede deberse a que en

Cuadro 2. Producción de biomasa por cosecha y anual del pasto alpiste y capacidad de carga animal.

\begin{tabular}{|c|c|c|c|c|}
\hline Tratamiento $^{1}$ & $\begin{array}{c}\text { Biomasa } \\
\left(\mathrm{kg} \cdot \mathrm{ha}^{-1} \cdot \operatorname{cosecha}^{-1} \mathrm{MS}\right)\end{array}$ & $\begin{array}{l}\text { Biomasa anual } \\
\left(\mathrm{kg} \cdot \mathrm{ha}^{-1} \mathrm{MS}\right)^{2}\end{array}$ & $\begin{array}{c}\text { Carga animal estable } \\
\left(\text { U.A.ha }{ }^{-1}\right)\end{array}$ & $\begin{array}{c}\text { Utilización del N } \\
\left(\mathrm{kg} \mathrm{MS} \mathrm{kg}^{-1} \mathrm{~N}\right)\end{array}$ \\
\hline 100 & 2956 & 15413 & 4,22 & 151 \\
\hline 200 & 3096 & 16144 & 4,42 & 81 \\
\hline 300 & 3347 & 17451 & 4,78 & 58 \\
\hline Promedio & 3133 & 16336 & 4,48 & 97 \\
\hline
\end{tabular}

1. Cada tratamiento corresponde al promedio de 20 muestras.

2. Utilizando un promedio de 5,21 cosechas anualmente. 
regiones altas del trópico se puede obtener más cosechas anuales en comparación con lugares de clima templado donde existe una estacionalidad marcada que hace que los pastos perennes entren en latencia a finales del otoño. El pasto alpiste mostró producciones de MS similares entre los 3 tratamientos por lo que la carga animal estable se mantuvo relativamente constante (Cuadro 2). La carga animal estable fue similar a la encontrada por Villalobos (2012) para el mismo pasto con 3 edades de cosecha.

La utilización del $\mathrm{N}$ en el pasto alpiste disminuyó un $61 \%$ al aplicar $300 \mathrm{~kg} \cdot \mathrm{ha}^{-1}$ con respecto a la dosis de $100 \mathrm{~kg} \cdot \mathrm{ha}^{-1}$, lo cual indica que dicho forraje no fue eficiente en extraer dicho nutriente del suelo al utilizar dosis mayores. Brink y Casler (2009) encontraron que la eficiencia en el uso del $\mathrm{N}$ en pastos perennes de clima templado se ve disminuida con dosis superiores a $135 \mathrm{~kg} \cdot \mathrm{ha}^{-1} \mathrm{~N}$, lo cual se traduce en poco o ningún retorno agronómico por cada unidad de $\mathrm{N}$ aplicada. Cherney y Cherney (2005) por su parte mencionan que el pasto alpiste tiene una limitada respuesta productiva a la aplicación de $\mathrm{N}$, por lo que dosis altas incrementan las pérdidas al ambiente (Jin et al. 2010). Las pérdidas de nutrientes del suelo incrementan la posibilidad de contaminación de mantos acuíferos cercanos a los sistemas pastoriles, mientras que se reduce el potencial de ser reciclados y utilizados posteriormente por el pasto (Brink y Casler 2009).

\section{Contenido de $\mathbf{N}$ y sus fracciones en el pasto alpiste}

La época de muestreo y la dosis de $\mathrm{N}$ afectaron el contenido de proteína del pasto alpiste (Cuadro 3). Pastos perennes de clima templado fertilizados con $225 \mathrm{~kg} \cdot \mathrm{ha}^{-1} \mathrm{~N}$ en forma fraccionada han mostrado valores de PC similares (11,70-16,90\%) a los del pasto alpiste (Cherney y Cherney 2005). El pasto alpiste tuvo contenidos de PC similares con las 3 dosis de $\mathrm{N}$ evaluadas y con capacidad de llenar los requerimientos del ganado lechero especializado en regiones altas del trópico (NRC 2001).

Cuadro 3. Contenido de nitrógeno en el pasto alpiste con 3 dosis de fertilización.

\begin{tabular}{|c|c|c|c|c|c|c|c|}
\hline \multirow{2}{*}{ Tratamiento $^{1}$} & \multirow{2}{*}{ PC (\%) } & \multirow{2}{*}{$\begin{array}{l}\text { kg.ha-1 } \\
\text { año }^{-1} \mathrm{~N}\end{array}$} & \multicolumn{5}{|c|}{ Fracciones $^{2}$} \\
\hline & & & $\mathrm{A}(\%)$ & $\mathrm{B}_{1}(\%)$ & $\mathrm{B}_{2}(\%)$ & $\mathrm{B}_{3}(\%)$ & $\mathrm{C}(\%)$ \\
\hline 100 & 16,84 & 403 & $6,63^{\mathrm{a}}$ & $1,24^{\mathrm{c}}$ & $4,72^{\mathrm{ab}}$ & $3,87^{\mathrm{a}}$ & 1,95 \\
\hline 200 & 16,44 & 416 & $5,25^{\mathrm{b}}$ & $1,96^{\mathrm{b}}$ & $4,74^{\mathrm{a}}$ & $3,24^{\mathrm{b}}$ & 1,82 \\
\hline 300 & 16,86 & 453 & $5,28^{\mathrm{b}}$ & $2,61^{\mathrm{a}}$ & $3,96^{\mathrm{b}}$ & $3,35^{\mathrm{ab}}$ & 1,99 \\
\hline Tratamiento $(\mathrm{T})$ & 0,6560 & - & 0,0431 & 0,0032 & 0,1137 & 0,0849 & 0,4954 \\
\hline Muestreo (M) & $<0,0001$ & - & 0,0006 & $<0,0001$ & 0,0001 & $<0,0001$ & 0,0002 \\
\hline $\mathrm{T} * \mathrm{M}$ & 0,0053 & - & 0,0733 & 0,0081 & 0,1988 & 0,2598 & 0,2356 \\
\hline
\end{tabular}

1. Cada tratamiento corresponde al promedio de 11 muestras.

2. Medias con letras diferentes en una columna difieren entre si según la prueba de Duncan $(\mathrm{p}<0,05)$.

Por medio del contenido de proteína y la biomasa producida, se estimó la capacidad de extracción de $\mathrm{N}$ del suelo del pasto alpiste, la cual aumentó en $50 \mathrm{~kg} \cdot \mathrm{ha}^{-1}$ al incrementar la dosis de $\mathrm{N}$ de 100 a $300 \mathrm{~kg} \cdot \mathrm{ha}^{-1}$. Lo anterior indica que el pasto alpiste utilizó una pequeña cantidad del $\mathrm{N}$ aplicado con dosis mayores a $100 \mathrm{~kg} \cdot \mathrm{ha}^{-1}$ $\mathrm{N}$. En general, el $\mathrm{N}$ ha mostrado potencial para incrementar el contenido de proteína y nutrientes digestibles totales en pastos, sin embargo, sus 
efectos varían de acuerdo con la dosis empleada, la especie/cultivar y diferencias climatológicas (Funderburg et al. 2012). Cherney y Cherney (2005) reportaron extracciones de $\mathrm{N}$ del pasto alpiste de $187 \mathrm{~kg}$ ha $^{-1}$ durante la época de crecimiento (mayo-noviembre) en condiciones de clima templado. En el presente estudio se obtuvo extracciones de $\mathrm{N}$ superiores, lo cual puede deberse a que en regiones altas del trópico la producción de biomasa de los pastos se mantiene relativamente constante a lo largo del año, cuando se mostraron cambios fueron debidos principalmente al régimen de precipitación.

Las fracciones de la PC del pasto alpiste se vieron afectadas principalmente por la época de muestreo, mientras que la interacción entre la época de muestreo y la dosis de $\mathrm{N}$ fue significativa para las fracciones A y $\mathrm{B}_{1}(\mathrm{p}=0,0242$ y 0,0038 , respectivamente) (Cuadro 3 ). La fracción de $\mathrm{N}$ soluble (A) disminuyó conforme se aumentó la dosis de $\mathrm{N}$, y fue menor en la dosis de $100 \mathrm{~kg} \cdot \mathrm{ha}^{-1}$. La fracción $\mathrm{B}_{1}$ aumentó igualmente con la dosis de $\mathrm{N}$; dicha fracción se compone de péptidos de menor tamaño y es de mayor importancia en pastos de clima templado
(WingChing y Rojas-Bourrilon 2006). Las fracciones $\mathrm{B}_{2}, \mathrm{~B}_{3}$ y $\mathrm{C}$ tuvieron contenidos similares con las 3 dosis de $\mathrm{N}$.

Las fracciones del $\mathrm{N}$ degradable a nivel de rumen $\left(A+B_{1}\right)$, insoluble degradable (proteína de sobrepaso; $\mathrm{B}_{2}+\mathrm{B}_{3}$ ) e indigerible $(\mathrm{C})$ en el pasto alpiste fueron en promedio 43,4, 45,6 y 11,0\%, respectivamente. Con base en lo anterior, el pasto alpiste fertilizado tiene potencial de proveer cerca del $50 \%$ de las fracciones de proteína utilizable a nivel de rumen, mientras que las fracciones restantes $\mathrm{B}_{2}, \mathrm{y}_{3}$ sobrepasan la degradación ruminal para su posterior utilización a nivel de intestino.

\section{Contenido de carbohidratos y digestibilidad del pasto alpiste}

El contenido de pared celular (FDN) y la digestibilidad (DIVMS) del pasto alpiste mostraron cambios significativos debidos a la época de muestreo mientras que la dosis de $\mathrm{N}$ no afectó su contenido (Cuadro 4). La pared celular y la lignina aumentaron de febrero a agosto y disminuyeron en noviembre; mostraron así mismo la misma tendencia para las 3 dosis de $\mathrm{N}$ evaluadas (Figura 3). El contenido de pared

Cuadro 4. Contenido de pared celular, carbohidratos no fibrosos y digestibilidad in- vitro de la MS del pasto alpiste a lo largo de un año.

\begin{tabular}{|c|c|c|c|c|}
\hline Muestreo $^{1}$ & $\mathrm{FDN}^{2}(\%)$ & Lignina (\%) & $\mathrm{CNF}(\%)$ & DIVMS (\%) \\
\hline Feb-02 & $54,5^{\mathrm{d}}$ & $2,1^{\mathrm{b}}$ & $15,8^{\mathrm{a}}$ & $57,8^{\mathrm{b}}$ \\
\hline Abr-13 & $63,3^{\mathrm{c}}$ & $2,0^{\mathrm{b}}$ & $11,3^{\mathrm{b}}$ & $69,7^{\mathrm{a}}$ \\
\hline Jun-22 & $66,1^{\mathrm{b}}$ & $3,9^{\mathrm{a}}$ & $8,1^{\mathrm{c}}$ & $43,2^{\mathrm{c}}$ \\
\hline Ago-31 & $70,7^{\mathrm{a}}$ & $4,2^{\mathrm{a}}$ & $3,4^{\mathrm{d}}$ & $44,5^{\mathrm{c}}$ \\
\hline Nov-11 & $62,1^{\mathrm{c}}$ & $2,5^{\mathrm{b}}$ & $10,4^{\mathrm{b}}$ & $59,8^{\mathrm{b}}$ \\
\hline Promedio & 63,3 & 2,9 & 9,8 & 55,0 \\
\hline Tratamiento $(\mathrm{T})$ & 0,9346 & 0,8552 & 0,0440 & 0,8473 \\
\hline Muestreo (M) & $<0,0001$ & $<0,0001$ & $<0,0001$ & $<0,0001$ \\
\hline $\mathrm{T} * \mathrm{M}$ & 0,2466 & 0,6282 & 0,3528 & 0,2175 \\
\hline
\end{tabular}

1. Cada muestreo corresponde al promedio de las 12 parcelas.

2. Medias con letras diferentes en una columna difieren entre si según la prueba de Duncan $(\mathrm{p}<0,05)$. 


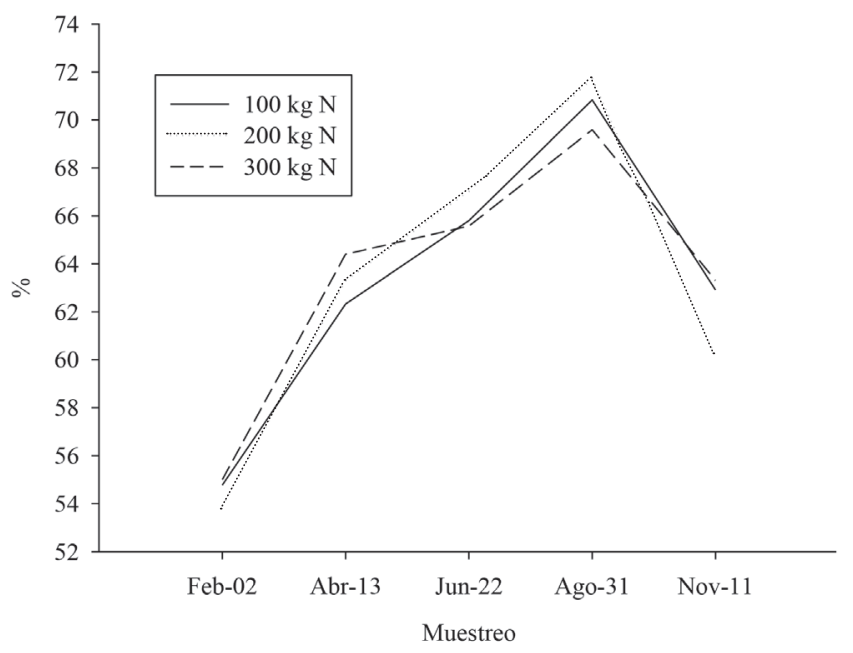

Fig. 3. Contenido de pared celular del pasto alpiste con 3 dosis de $\mathrm{N}$ a lo largo de 5 muestreos en zonas altas del trópico $\mathrm{N}$ a lo largo de 5 muestreos en zonas altas del trópico.

celular promedio del pasto alpiste fue mayor al encontrado en previos estudios donde se evaluó 3 edades de cosecha $(53,18-57,77 \%)$ (Villalobos 2012) y 3 dosis de N (51,70-53,40\%) (Cherney y Cherney 2005). El contenido de lignina fue menor a lo reportado por Villalobos (2012) con $3,78-3,42 \%$ para el pasto alpiste y similar al de los pastos ryegrass perenne y kikuyo en pastoreo (Andrade 2006, Villalobos y Sánchez 2010b).

El contenido de carbohidratos no fibrosos del pasto alpiste mostró cambios significativos debidos a la dosis de $\mathrm{N}(\mathrm{p}=0,0001)$ y la época de muestreo ( $\mathrm{p} \leq 0,0001)$ (Cuadro 4). Los CNF disminuyeron en junio y agosto, lo cual puede deberse al incremento en pared celular en dicha época. La radiación solar en la zona donde se realizó el estudio es menor de mayo a noviembre (3,1-4,2 horas.día $\left.{ }^{-1}\right)$ en comparación al periodo de diciembre a abril (6,6-8,8 horas.día ${ }^{-1}$ ) (IMN 2015). Sin importar la región climática, la radiación solar es uno de los componentes básicos que influye sobre la tasa de fotosíntesis en pastos (Donaghy y Fulkerson
2002) por lo que una radiación solar menor con una respiración similar, puede disminuir el nivel de reservas de carbohidratos en la planta.

La digestibilidad del pasto alpiste varió significativamente $(\mathrm{p} \leq 0,0001)$ a lo largo del año, lo cual podría impactar la productividad del ganado lechero especializado en regiones altas del trópico (Cuadro 4). La digestibilidad disminuyó en junio y agosto, lo cual se pudo deber a que el contenido de fibra aumentó y el de carbohidratos no fibrosos disminuyó en los mismos meses. La DIVMS del pasto alpiste fue menor a la de pastos utilizados en regiones altas del trópico (Andrade 2006, Villalobos y Sánchez 2010b) y similar a la de pastos tropicales como estrella africana (Villalobos y Arce 2014). El pasto alpiste ha mostrado valores de digestibilidad in-vitro verdadera (DIVV) y de digestibilidad in-vitro de la materia orgánica (DIVMO) mayores a los encontrados en este estudio (78,5-85,2\% y 60,0$85,0 \%$, respectivamente) (Geber 2002, Cherney y Cherney 2005). 
Las muestras de pasto alpiste en este estudio se cosecharon a una altura menor a la utilizada cuando se toman muestras en sistemas de pastoreo, en donde primordialmente el componente foliar es removido por los animales y, que a su vez, se trata de simular al momento de recolectar muestras (Donaghy y Fulkerson 1997). La disminución en digestibilidad del pasto alpiste entre abril y junio (23 puntos porcentuales) puede tener un impacto significativo en el consumo de MS y la producción láctea (NRC 2001), por lo que un programa de suplementación estratégica permitiría llenar los nutrientes limitantes del pasto durante ciertas épocas del año.

\section{Contenido energético del pasto alpiste}

Las 3 dosis de $\mathrm{N}$ evaluadas no afectaron el contenido energético del pasto alpiste mientras que hubo diferencias significativas $(p \leq 0,0001)$ entre los muestreos durante el año (Cuadro 5). El total de nutrientes digestibles (TND) fue mayor en febrero y disminuyó gradualmente hasta alcanzar el contenido menor en agosto (Figura 4). Dicho comportamiento se relaciona directamente con el aumento en fibra (Figura 3) y la disminución en carbohidratos no fibrosos (Cuadro 4) que el pasto alpiste mostró en agosto.

Cuadro 5. Contenido de energía del pasto alpiste a lo largo de un año.

\begin{tabular}{lcc}
\hline Muestreo $^{1}$ & $\begin{array}{c}\mathrm{TND}^{2} \\
(\%)\end{array}$ & $\begin{array}{c}\mathrm{EN}_{\mathrm{L}} \\
\left(\mathrm{Mcal}^{-1} \mathrm{~kg}^{-1} \mathrm{MS}^{2}\right.\end{array}$ \\
\hline Feb-02 & $63,2^{\mathrm{a}}$ & $1,32^{\mathrm{a}}$ \\
Abr-13 & $61,3^{\mathrm{b}}$ & $1,26^{\mathrm{b}}$ \\
Jun-22 & $57,7^{\mathrm{c}}$ & $1,17^{\mathrm{c}}$ \\
Ago-31 & $55,5^{\mathrm{d}}$ & $1,14^{\mathrm{d}}$ \\
Nov-11 & $60,1^{\mathrm{b}}$ & $1,27^{\mathrm{b}}$ \\
Promedio & 59,6 & 1,23 \\
\hline Tratamiento $(\mathrm{T})$ & 0,7685 & 0,8515 \\
Muestreo $(\mathrm{M})$ & $<0,0001$ & $<0,0001$ \\
$\mathrm{~T}^{*} \mathrm{M}$ & 0,3094 & 0,8524 \\
\hline
\end{tabular}

1. Cada muestreo corresponde al promedio de las 12 parcelas.

2. Medias con letras diferentes en una columna difieren entre si según la prueba de Duncan $(\mathrm{p}<0,05)$. 


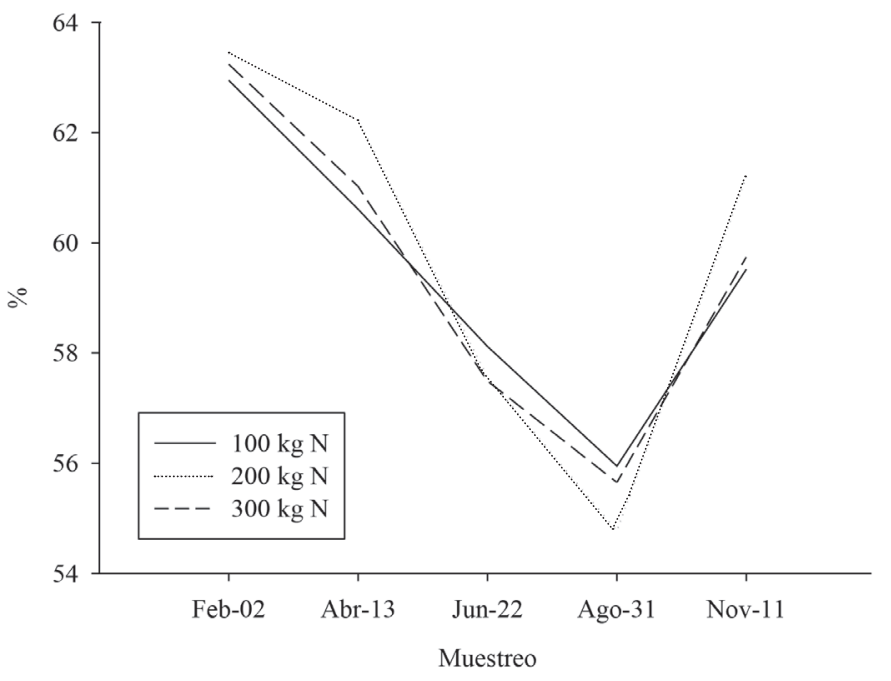

Fig. 4. Total de nutrientes digestibles del pasto alpiste con 3 dosis de N.

En promedio, el contenido de energía neta de lactancia $\left(E_{L}\right)$ en el pasto alpiste fue menor al reportado para pastos utilizados en regiones altas del trópico (Andrade 2006; Villalobos y Sánchez 2010b; Villalobos 2012). Pastos tropicales como estrella africana han mostrado contenidos de energía similares a los del pasto alpiste (Villalobos y Arce 2014). El contenido de energía en pastos es un indicador que integra los diferentes componentes nutricionales y permite estimar su potencial de producción láctea, utilizándose como base para el plan de suplementación en ganado lechero especializado. El pasto alpiste tuvo un contenido de energía relativamente bajo en comparación con pastos de clima templado (Villalobos y Sánchez 2010b). La producción del ganado lechero se puede ver limitada por el contenido de energía del pasto alpiste sino se brinda fuentes de energía de rápida disponibilidad a nivel ruminal que permitan una adecuada producción de proteína microbial.

\section{CONCLUSIONES Y RECOMENDACIONES}

El pasto alpiste tuvo producciones de biomasa similares con las 3 dosis de $\mathrm{N}$ evaluadas, y además se identificó que la época de muestreo fue el factor de mayor influencia sobre la cantidad producida. El nitrógeno se considera uno de los principales factores que permite incrementar la capacidad de carga en pasturas, sin embargo el pasto alpiste tuvo una limitada respuesta a dosis de $\mathrm{N}$ superiores a $100 \mathrm{~kg} \cdot \mathrm{ha}^{-1}$, por lo que su capacidad de carga se mantuvo relativamente constante.

Los programas de fertilización nitrogenada en pasto alpiste deben prever los cambios estacionales en producción de biomasa del pasto alpiste por influencia climatológica del caribe. El fraccionamiento de las dosis debe optimizar el uso del $\mathrm{N}$ en las épocas que el pasto tiene un potencial mayor de utilización. La cantidad de $\mathrm{N}$ a aplicar debe reducirse de noviembre a febrero pues el potencial productivo del pasto se vio 
limitado debido a factores climatológicos (humedad en el suelo y temperatura ambiental).

Según la fuente de $\mathrm{N}$ utilizada, el fraccionamiento de la dosis a aplicar puede generar un ahorro para el productor y menores pérdidas debidas a lixiviación y volatilización, lo cual a su vez disminuye la contaminación a mantos acuíferos y aumento de gases de efecto invernadero al ambiente. El pasto alpiste debe evaluarse en otras condiciones climatológicas, así como su respuesta productiva a otras fuentes de $\mathrm{N}$ de uso común tal como la Urea de lenta liberación en el suelo, Urea azufrada y el Nitrato de Amonio.

La fenología del pasto alpiste no sufrió cambios por efecto de la cantidad de $\mathrm{N}$ aplicada mientras que el número de hojas verdes por brote aumentó en las épocas en las que la producción de biomasa fue mayor. El pasto alpiste debe cosecharse cuando su edad fenológica sea al menos 5 hojas verdes.brote ${ }^{-1}$ (Villalobos 2012) con lo cual se garantice una adecuada recuperación de reservas en la planta.

Las dosis de $\mathrm{N}$ utilizadas no produjeron cambios significativos en el valor nutricional del pasto alpiste, sin embargo, las variaciones encontradas en el contenido de carbohidratos estructurales y no estructurales, la digestibilidad y la energía, pueden reducir la producción láctea en regiones altas del trópico. Los programas de suplementación de ganado lechero deben proveer mayor cantidad de nutrientes digestibles de junio a agosto pues el valor nutricional del pasto no logra llenar los requerimientos del ganado lechero.

\section{AGRADECIMIENTOS}

El autor expresa su agradecimiento al propietario de la finca donde se realizó esta investigación: Ing. Alvaro Coto, así como a los trabajadores de la finca por la colaboración brindada durante el tiempo en que se realizó la investigación.

\section{LITERATURA CITADA}

Andrade, M. 2006. Evaluación de técnicas de manejo para mejorar la utilización del pasto kikuyo (Pennisetum clandestinum Hochst. ex Chiov) en la producción de ganado lechero en Costa Rica. Tesis Lic. San José, Costa Rica, Universidad de Costa Rica. 225 p.

AOAC (Association of Official Analytical Chemist, USA). 1990. Official methods of analysis. 15th ed, Arlington, VA. 1008 p.

Brink, GE; Casler, MD. 2009. Meadow Fescue, Tall Fescue, and Orchardgrass Response to Nitrogen Application Rate. Forage and Grazinglands 7(1):1-12.

Cherney, DJR; Cherney, JH. 2005. Forage Yield and Quality of Temperate Perennial Grasses as Influenced by Stubble Height. Forage and Grazinglands 3(1):1-8.

Donaghy, DJ; Fulkerson, WJ. 1997. The importance of watersoluble carbohydrate reserves on regrowth and root growth of Lolium perenne (L.). Grass and Forage Science 52:401-407.

Donaghy, DJ; Fulkerson WJ. 2002. The impact of defoliation frequency and nitrogen fertilizer application in spring on summer survival of perennial ryegrass under grazing in subtropical Australia. Grass and Forage Science 57:351-359.

Funderburg, E; Biermacher, JT; Moffet, CA; Alkire, D; Mosali, J. 2012. Effects of Applying Five Nitrogen Rates on Quality of Nine Varieties of Introduced Perennial Forages. Forage and Grazinglands 10(1):1-14.

Geber, U. 2002. Cutting frequency and stubble height of reed canary grass (Phalaris arundinacea L.): influence on quality and quantity of biomass for biogas production. Grass and Forage Science 57:389-394.

IMN (Instituto Meteorológico Nacional, Costa Rica). 2015. Informe Annual. Climatología de la región del distrito de Santa Rosa de Oreamuno en base a estaciones meteorológicas cercanas a la zona. San José, Costa Rica. 3 p.

Janzen, DH. 1991. Historia Natural de Costa Rica. $1^{\text {a }}$ ed Editorial de la UCR, San José, Costa Rica. 822 p.

Jin, T; Shimizu, M; Marutani, S; Desyatkin, AR; Iizuka, N; Hata, H; Hatano, R. 2010. Effect of chemical fertilizer and manure application on $\mathrm{N}_{2} \mathrm{O}$ emission from reed canary grassland in Hokkaido, Japan. Soil Science \& Plant Nutrition 56:53-65.

Licitra, G; Hernandez, TM; Van Soest, PJ. 1996. Standardization of procedures for nitrogen fractionation of ruminant feeds. Animal Feed Science and Technology 57:347-358.

Mandaluniz, N; Ruiz, R; Oregui, LM. 2005. Propuesta de definición de unidad animal y metodología de estimación para su aplicación en sistemas de pastoreo extensivo. In XLV Reunión Científica de 
la SEEP (Sesión: Sistemas Agrosilvopastorales). Asturias, España. p. 275-280.

NRC (National Research Council, USA). 2001. Nutrient Requirements of Dairy Cattle: Seventh Revised Edition, 2001. The National Academies Press, Washington, DC. USA. 381 p.

SAS (SAS-Institute). 2011. The SAS system for Windows $\mathrm{N}^{\circ}$. 9.3. SAS Institute, Cary, NC.

Van Soest, PJ; Robertson, JB. 1985. Analysis of forages and fibrous foods: a laboratory manual for animal science. Cornell University, Ithaca, NY. p. 202.

Van Soest, PJ; Robertson, JB; Lewis, BA. 1991. Methods for Dietary Fiber, Neutral Detergent Fiber, and Nonstarch Polysaccharides in Relation to Animal Nutrition. Journal of Dairy Science 74:3583-3597.

Villalobos, L. 2012. Fenología, producción y valor nutritivo del pasto alpiste (Phalaris arundinacea) en la zona alta lechera de Costa Rica. Agronomía Costarricense 36:25-37.
Villalobos, L; Arce, J. 2014. Evaluación agronómica y nutricional del pasto estrella africana (Cynodon nlemfuensis) en la zona de Monteverde, Puntarenas, Costa Rica. II . Valor nutricional. Agronomía Costarricense 38:133-145.

Villalobos, L; Sánchez, JM. 2010a. Evaluación agronómica y nutricional del pasto ryegrass perenne tetraploide (Lolium perenne) producido en lecherías de las zonas altas de Costa Rica. I. Producción de biomasa y fenología. Agronomía Costarricense 34:31-42.

Villalobos, L; Sánchez, JM. 2010b. Evaluación agronómica y nutricional del pasto ryegrass perenne tetraploide (Lolium perenne) producido en lecherías de las zonas altas de Costa Rica. I. Valor nutricional. Agronomía Costarricense 34:43-52.

WingChing, R; Rojas-Bourrilon, A. 2006. Composición nutricional y características fermentativas del ensilaje de maní forrajero. Agronomía Costarricense $30: 87-100$. 
